\title{
Fatty acid synthesis is a therapeutic target in human liposarcoma
}

\author{
ARNE M. OLSEN ${ }^{1}$, BURTON L. EISENBERG ${ }^{2,3}$, NANCY B. KUEMMERLE ${ }^{3,5}$, ALISON J. FLANAGAN AL, $^{3,4}$ \\ PETER M. MORGANELLI ${ }^{3,6}$, PORTIA S. LOMBARDO ${ }^{3,4}$, JOHANNES V. SWINNEN ${ }^{7}$ and WILLIAM B. KINLAW ${ }^{3,4}$ \\ ${ }^{1}$ Department of Surgery, Dartmouth-Hitchcock Medical Center, Lebanon; ${ }^{2}$ Department of Surgery, \\ Section of Surgical Oncology, Dartmouth-Hitchcock Medical Center, Lebanon, New Hampshire and Dartmouth \\ Medical School, Hanover; ${ }^{3}$ Norris Cotton Cancer Center at Dartmouth-Hitchcock Medical Center; Departments of \\ ${ }^{4}$ Medicine, Section of Endocrinology and Metabolism, and ${ }^{5}$ Medicine, Section of Hematology and Oncology, \\ Dartmouth-Hitchcock Medical Center, Lebanon, New Hampshire and Dartmouth Medical School; ${ }^{6}$ Department of \\ Immunology and Microbiology, Dartmouth Medical School, Lebanon, NH, USA; ${ }^{7}$ Laboratory for Experimental \\ Medicine and Endocrinology, Katholieke Universiteit Leuven, Leuven, Belgium
}

Received January 13, 2010; Accepted February 26, 2010

DOI: 10.3892/ijo_00000616

\begin{abstract}
Liposarcomas (LS) are mesenchymal tumors that can recur after surgical resection and often do not respond to presently available medical therapies. This study demonstrates the dependence of LS on de novo long-chain fatty acid synthesis for growth. Lipogenesis can be impaired by inhibiting the activities of lipogenic enzymes, including acetyl CoA-carboxylase (ACC) and fatty acid synthase (FASN), or by suppressing the expression of key genes involved in the pathway and its regulation. The FASN inhibitors cerulenin and orlistat reduced the growth of two LS cell lines (LiSa2, SW872), as did inhibition of ACC with soraphen A. CDDO$\mathrm{Me}$, a synthetic triterpenoid, suppressed expression of Spot 14 and FASN genes and likewise inhibited LS cell growth. Importantly, the anti-proliferative effect of each agent was prevented by the co-administration of palmitate, the major product of cellular long-chain fatty acid synthesis. In stark contrast to LS cells, these compounds had no effect on the growth of fibroblasts. Four biochemically distinct agents that target critical points in the fatty acid synthetic pathway exert anti-proliferative effects on LS cells, and rescue of cell growth by palmitic acid suggests that reduced tumor cell lipogenesis mediates the growth inhibition. These findings warrant further studies aimed at the clinical exploitation of the dependence of LS cell growth on fatty acids.
\end{abstract}

Correspondence to: Dr William Kinlaw, 606 Rubin Building, Dartmouth Medical School, One Medical Center Drive, Lebanon, NH 03756, USA

E-mail: william.kinlaw@hitchcock.org

Abbreviations: ACC, acteyl CoA-carboxylase; CYC, cyclophilin; CDDO-Me, 2-cyano-3,12-dioxooleana-1,9-dien-28-oic methyl ester; DMSO, dimethyl sulfoxide; FASN, fatty acid synthase; FBS, fetal bovine serum; LS, liposarcoma; PCR, polymerase chain reaction

Key words: fatty acid synthase, liposarcoma, spot 14

\section{Introduction}

Liposarcomas (LS) are uncommon tumors with a yearly incidence of $\sim 5,000$ cases in the U.S. They are typically large extremity or retroperitoneal tumors with a high rate of recurrence following attempted surgical resection. Additionally, recurrent LS respond poorly to conventional medical therapies $(1,2)$. Molecular and morphologic properties of these tumor cells are similar to adipocytes, and it is likely that they arise from an adipogenic precursor cell. Not surprisingly, gene expression array analysis of a panel of LS revealed that a substantial number of up-regulated genes were involved in fatty acid metabolism (3). Our recent examination of available LS cell lines and tumor tissues indicates that this malignancy exhibits a lipogenic phenotype (4). Lipogenic malignancies depend on ongoing lipid synthesis for growth and survival, and this phenotype has been described in a variety of cancers including those of breast, ovary, colon, prostate and leukemia (5). The common genetic signature of lipogenic tumors is the upregulated expression of Spot 14 (THRSP) and lipogenic enzymes, exemplified by acetyl CoA-carboxylase (ACC) and fatty acid synthase (FASN). ACC carboxylates acetyl-CoA to yield malonyl CoA, while FASN catalyzes the multistep synthesis of palmitate from malonyl-CoA and acetyl-CoA (5). Spot 14, a nuclear protein associated with transcriptional activation of several enzymes of the fatty acid synthetic pathway, is also highly expressed in lipogenic tumors $(6,7)$. High levels of FASN and Spot 14 confer a survival advantage to tumor cells, and predict a poor prognosis in breast cancer $(5,6,8)$. Accordingly, there has been significant investigative interest in manipulating this metabolic pathway for therapeutic effect.

We previously demonstrated upregulated expression of lipogenesis-related genes, including FASN and Spot 14, in LiSa-2 human LS cells (4). We now report a similar pattern in the SW872 line, and further investigated LiSa-2 cells to assess the potential of targeting lipid metabolism using four structurally distinct compounds with different modes of action to impair lipogenesis: cerulenin, orlistat, soraphen A, 
and the synthetic triterpenoid CDDO-Me. Cerulenin, a natural product of Cephalosporium caerulens, inhibits FASN activity by irreversibly binding the $\beta$-ketoacyl-acyl carrier protein site of the enzyme, and induces apoptosis in a variety of lipogenic human cancer cell lines (9). Orlistat is also a FASN inhibitor with antitumor activity that binds to the thioester domain of the enzyme $(10,11)$. Soraphen A is a potent inhibitor of acetyl CoA-carboxylase (ACC) enzyme activity (12). Synthetic triterpenoids are derivatives of the naturally occurring triterpenoids, oleanolic and urosolic acid. These agents manifest a variety of activities in different cell lines, including anti-proliferative, anti-inflammatory and prodifferentiation effects (13). We previously showed that a triterpenoid downregulates FASN and Spot 14 gene expression in LiSa-2 LS cells (4). We now have confirmed this finding using a quantitative assay, and further show that the attendant growth inhibition is prevented by administration of palmitate and that fibroblasts are not affected by the triterpenoid. Our findings support the overall hypothesis that targeted interruption of de novo fatty acid synthesis by each of these distinct anti-lipogenic agents in LS cell lines inhibits growth and appears to be specific for lipogenic, malignant cells. Moreover, reversal of these growth inhibitory effects by provision of palmitate in each case indicates that most or all of the observed tumor cell anti-proliferative effect is mediated by depletion of cellular fatty acids.

\section{Materials and methods}

Reagents. Cerulenin (Sigma, St. Louis, MO) and CDDO-Me (manufactured under the NIH RAID Program) were prepared in dimethyl sulfoxide (DMSO). Orlistat was a kind gift from Roche, and soraphen A was kindly provided by Drs Rolf Jansen and Klaus Gerth (Helmholtz-Zentrum für Infektionsforschung, Braunschweig, Germany). Both inhibitors were solublized in ethanol. Palmitic acid (Sigma) was solublized using delipidated albumin (Sigma) as described by Ip and coworkers (14).

Cell lines and media. The LiSa-2 liposarcoma cells were kindly provided by Martin Wabitsch (University of Ulm, Germany) (15), SW872 cells were from American Type Culture Collection (Manassas, VA), and human fibroblasts were a kind gift from Eva Rzucidlo (Dartmouth Medical School, Hanover, NH). All cells were of low $(<10)$ passage number following resuscitation of frozen stocks. The identity of liposarcoma cells was verified by the appearance of typical cytosolic lipid droplets following confluence and exposure to adipogenic medium. Cells were grown in DMEM/Ham's F12 50:50 (Mediatech Inc, Herndon, VA) media supplemented with 10\% FBS (Atlanta Biologicals, Lawrenceville, GA), 1\% penicillin-streptomycin solution (Mediatech Inc) and $1 \%$ L-glutamine (Mediatech Inc). Adipogenic media also contained $1 \mathrm{nM}$ insulin, $20 \mathrm{pM}$ triiodothyronine and $1 \mu \mathrm{M}$ hydrocortisone (Sigma). Cells were cultured on plastic at $37^{\circ} \mathrm{C}$ in $4.5 \% \mathrm{CO}_{2}$.

Evaluation of gene expression. The Qiagen RNeasy kit (Valencia, CA) was used to extract RNA. RNA was shown to be intact by gel electrophoresis and to be free of contaminating

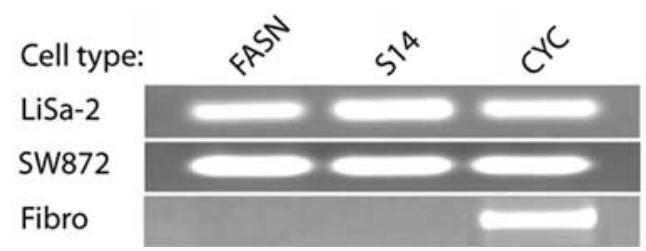

Figure 1. Lipogenesis-related RT-PCR gene profile for liposarcoma cell lines. An ethidium-stained agarose gel electrophoresis of RT-PCR products after 25 cycles is shown. Primers for fatty acid synthase (FASN), Spot 14 (S14) and cyclophilin (CYC) were used to analyze two different liposarcoma cell lines (LiSa-2, SW872) and fibroblasts (Fibro). Template mRNA was harvested after induction of differentiation in the liposarcoma cells.

DNA by the failure of polymerase chain reaction (PCR) to yield a product using primers corresponding to the cyclophilin gene. Random primers and reverse transcriptase were from New England Biolabs (Ipswich, MA). Qualitative PCR (25 cycles) was carried out using primers for FASN, Spot 14 and cyclophilin (CYC) as previously described (7). Quantitative real-time PCR (40 cycles) was carried out using the MyiQ Single Color real-time PCR detection system (BioRad, Hercules, CA) with SYBR-Green fluorescence (Applied Biosystems, Warrington, UK). Expression of specific mRNAs was expressed as a ratio to that of cyclophilin mRNA.

Lipid synthesis. Lipid synthesis was assessed by incubating cells in 1-[14-C]-acetate (Sigma; $20.8 \mathrm{mCi} / \mathrm{mmol}, 1 \mu \mathrm{Ci}$ in $200 \mu \mathrm{lmedium} /$ well) for $3 \mathrm{~h}$, followed by extraction by the method of Bligh and Dyer (16) and liquid scintillation spectrophotometry. Counting rates were corrected for the cellular protein content estimated by the method of Waddell (17).

Cell proliferation. Cells were seeded at a density of $10,000 / \mathrm{cm}^{2}$ in 48-well plates, and test media were added the next morning. Cells were treated for 5 days with appropriate vehicle controls in the presence or absence of $32 \mu \mathrm{M}$ palmitic acid. Media (200 $\mu \mathrm{l} /$ well) were replaced every $72 \mathrm{~h}$. Cerulenin concentrations were derived from our prior experiments (4). Dosages of orlistat $(20$ or $40 \mu \mathrm{M})$ were based on published work (10). Soraphen A was used at a concentration determined by preliminary dose-response experiments. CDDOMe was dosed at $100 \mathrm{nM}$ as in our prior investigations (4). The palmitic acid concentration of $32 \mu \mathrm{M}$ was derived from preliminary dose-response experiments (data not shown). DNA content/well was assessed using the Hoechst assay (18).

Statistics. Experiments were repeated at least once with $>6$ samples for each group. Error bars represent the standard error of the mean. Two-way analysis of variance (ANOVA) and post hoc analysis were used to determine the statistical significance of differences between means.

\section{Results}

Gene expression in LS cell lines. We evaluated FASN and Spot 14 gene expression using reverse transcriptase PCR. Each of the genes was expressed in both LS lines upon 

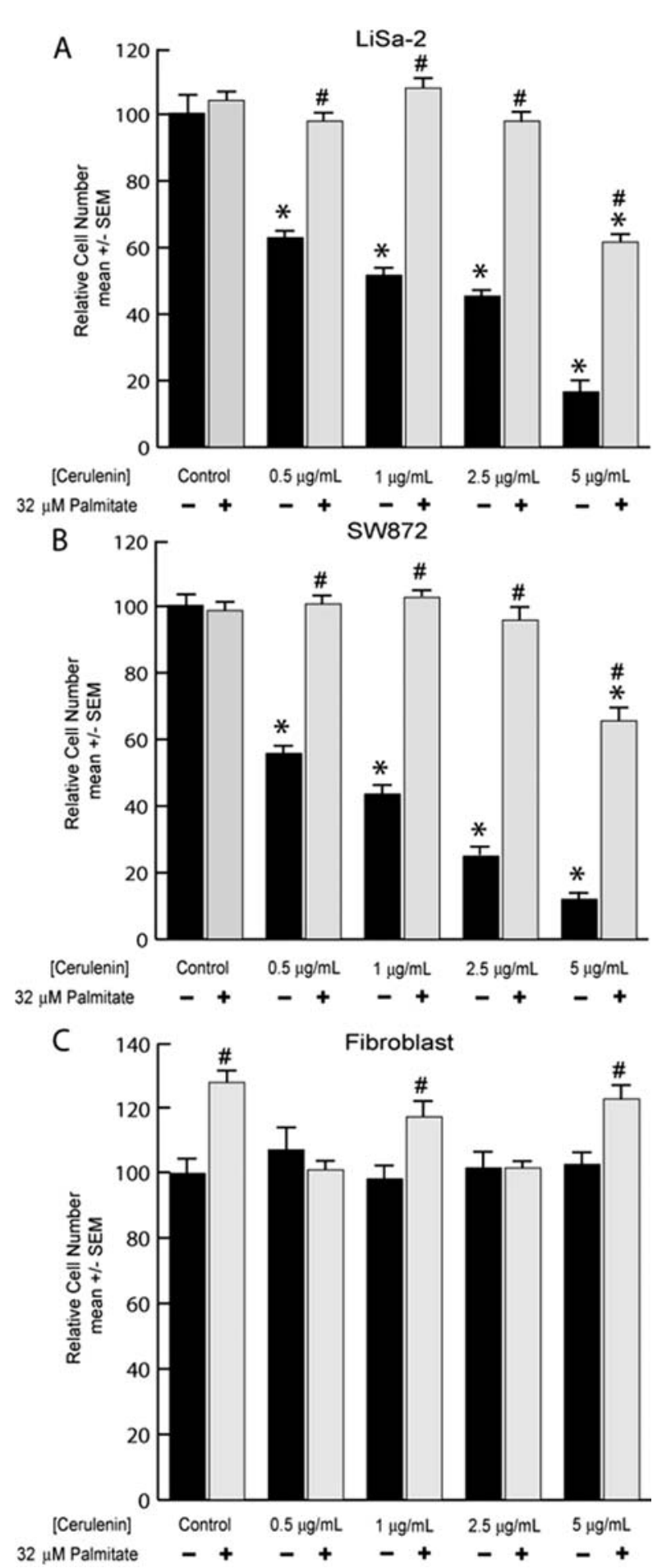

Figure 2. Anti-proliferative effect of cerulenin. (A) LiSa-2 cells were grown for 5 days in the indicated concentrations of cerulenin with or without $32 \mu \mathrm{M}$ palmitate. DNA content/well was assessed using the Hoechst assay. (B and C) Replicated experiment in SW872 and fibroblast cells. Data are mean \pm SEM normalized to the control group; $\mathrm{n}=8$ /group. ${ }^{*} \mathrm{p}<0.0001$ comparing cerulenin treatment to control; ${ }^{\#} \mathrm{p}<0.03$ comparing palmitate with cerulenin to corresponding cerulenin dose alone.

induction of differentiation with adipogenic media, whereas variable expression levels were observed beforehand (Fig. 1). In contrast, FASN and Spot 14 mRNAs were not detectable in fibroblasts after 25 cycles of PCR. Thus, as we previously demonstrated for LiSa-2 cells (4), SW872 LS cells exhibit adipocyte-like expression of these lipogenesis-related genes.
A

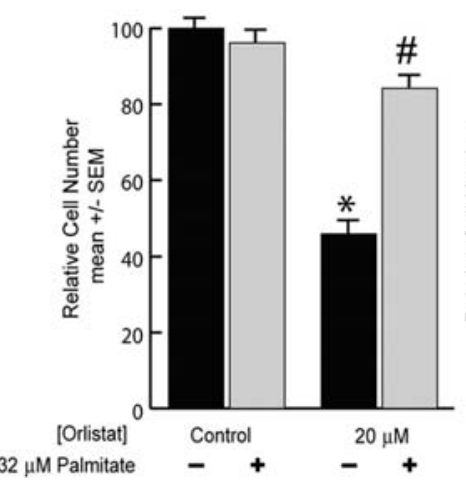

B Fibroblast

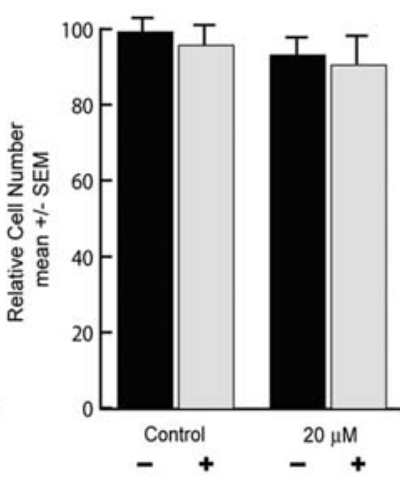

Figure 3. Anti-proliferative effect of orlistat. (A) LiSa-2 cells were grown for 5 days in vehicle or $20 \mu \mathrm{M}$ orlistat in the presence or absence of $32 \mu \mathrm{M}$ palmitate. DNA content/well was assessed using the Hoechst assay. (B) Replicated experiment in fibroblasts. DNA content/well was assessed using the Hoechst assay. Data are mean \pm SEM normalized to the control group; $\mathrm{n}=8$ /group. ${ }^{*} \mathrm{p}<0.0001$ comparing orlistat treatment to control; ${ }^{*} \mathrm{p}<0.000001$ comparing palmitate with orlistat to the corresponding orlistat dose alone.

Effects of cerulenin. Based on a dose-response data for LiSa-2 cell growth demonstrated in our prior work, we used cerulenin concentrations of $0.5-5 \mu \mathrm{g} / \mathrm{ml}$, with or without $32 \mu \mathrm{M}$ palmitic acid (4). We confirmed our previous dose-response for cerulenin in LiSa-2 cells (Fig. 2A). Increasing doses of cerulenin produced stepwise reductions in DNA content per well, with an $84 \%$ reduction at $5 \mu \mathrm{g} / \mathrm{ml}$ cerulenin $(\mathrm{p}<0.0001)$. We observed a rescue from cerulenin-induced growth inhibition when $32 \mu \mathrm{M}$ palmitic acid was provided to the cells. A significant $(\mathrm{p}<0.01)$ rescue effect was noted at $5 \mu \mathrm{g} / \mathrm{ml}$ cerulenin, with a return to $61 \%$ of control DNA content per well. Further significant recuperative effects, with a full return to control growth rates, were noted at each of the lower cerulenin concentrations.

SW872 cells responded similarly to the LiSa-2 cells, exhibiting significant reductions in DNA content per well at each of the cerulenin doses (Fig. 2B). Moreover, SW872 cells also demonstrated a restoration of normal growth upon provision of palmitate in the presence of cerulenin. Growth in the presence of the highest $(5 \mu \mathrm{g} / \mathrm{ml})$ cerulenin dose returned to $68 \%$ of control levels, while a complete rescue was again observed at lower concentrations. Fibroblasts (Fig. 2C) did not exhibit any significant reduction in DNA content per well across the entire cerulenin dosing regimen $(\mathrm{p}=0.64)$. In contrast to LS cells, some fibroblasts displayed a small augmentation $(\mathrm{p}<0.01)$ of DNA content per well in response to palmitate. Given the comparable results in both LS lines, we focused on the LiSa- 2 cell line in subsequent studies.

Effects of orlistat. We treated LiSa-2 and fibroblast cells with two different concentrations of the FASN enzyme inhibitor orlistat. In the LiSa-2 cells, we observed a significant $54 \%$ decrease $(\mathrm{p}<0.00001)$ in DNA content per well at $20 \mu \mathrm{M}$ orlistat. When the orlistat treatment was accompanied by $32 \mu \mathrm{M}$ palmitic acid, the DNA content per well was nearly restored to control levels (Fig. 3A). The fibroblasts did not exhibit any significant response to either orlistat or palmitic acid (Fig. 3B; p=0.58). 

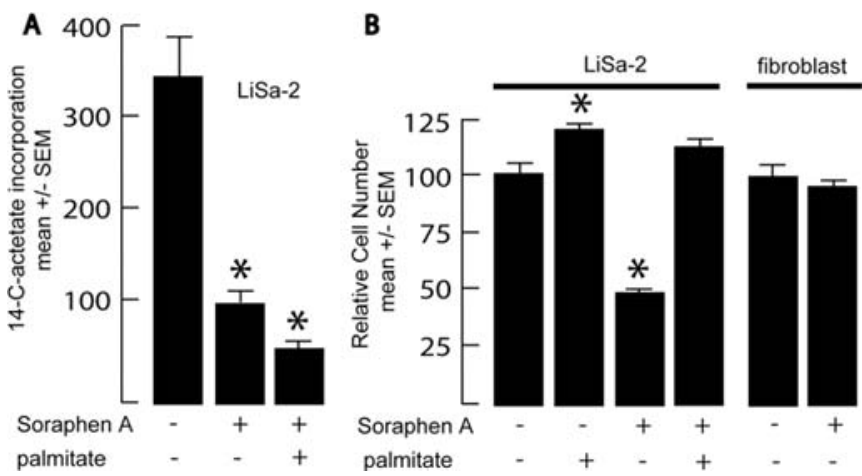

Figure 4. Effects of soraphen A. (A) LiSa-2 cells were grown for $24 \mathrm{~h}$ in vehicle or $75 \mathrm{nM}$ soraphen A with or without $32 \mu \mathrm{M}$ palmitate, at which time lipid synthesis (cpm incorporated/mg protein) was assessed over a $3-\mathrm{h}$ labeling period with 1-[14-C]-actetate. (B) LiSa-2 cells were grown for 5 days in $75 \mathrm{nM}$ soraphen $\mathrm{A}$ in the presence or absence of $32 \mu \mathrm{M}$ palmitate, and DNA content/well was assessed using the Hoechst assay. Data are mean \pm SEM with $\mathrm{n}=8$ /group. ${ }^{*} \mathrm{p}<0.01$ compared to vehicle control.

A

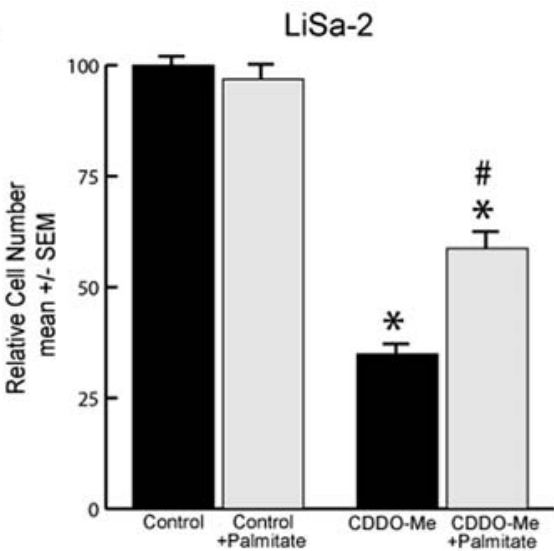

B

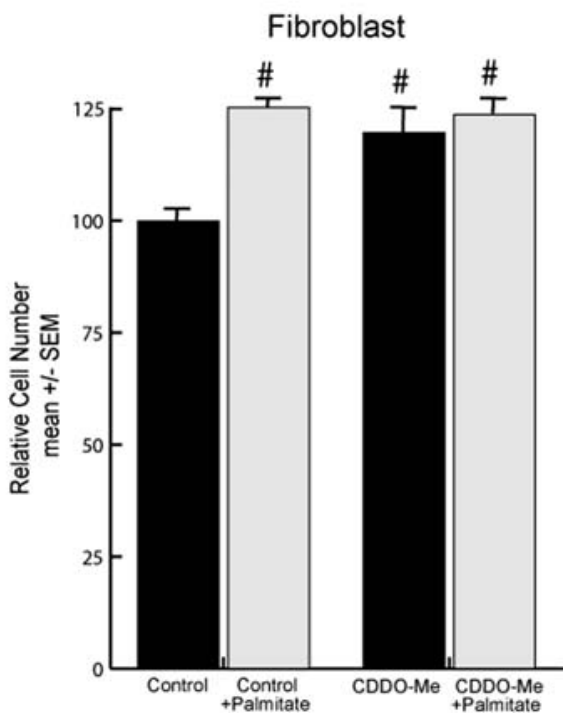

Figure 5. Anti-proliferative effects of CDDO-Me. (A) LiSa-2 cells were grown for 12 days in $100 \mathrm{nM}$ CDDO-Me in the presence or absence of $32 \mu \mathrm{M}$ palmitate. DNA content/well was assessed using the Hoechst assay. (B) Replicated experiment in fibroblasts. Data are expressed as mean \pm SEM with $n=8 /$ group normalized to the control groups. ${ }^{*} \mathrm{p}<0.001$ comparing CDDO-Me treatment to control; ${ }^{\#} \mathrm{p}<0.05$ comparing palmitate with CDDOMe to CDDO-Me alone.
Effects of soraphen A. Preliminary dose-response experiments indicated that $75 \mathrm{nM}$ soraphen A caused a significant growth inhibition of LiSa- 2 cells after $72 \mathrm{~h}$ (data not shown). In order to determine if this dose inhibited lipogenesis in LS cells, we assessed the incorporation of 14-C-actetate into lipids after $24 \mathrm{~h}$ exposure to the inhibitor (Fig. 4A). This resulted in a significant reduction in the rate of lipid synthesis to $29 \%$ of that observed in cells exposed to ethanol vehicle. Lipid synthesis was further reduced by co-administration of palmitate, perhaps indicating negative feedback of the pathway by its product. After $72 \mathrm{~h}$ exposure to the inhibitor, growth was significantly reduced in cells treated with soraphen A, and this effect was prevented by the co-administration of palmitic acid (Fig. 4B). Palmitic acid alone caused a small increase in Lisa- 2 cell growth in these experiments.

Effects of CDDO-Me. Treatment of LiSa-2 cells with $100 \mathrm{nM}$ CDDO-Me resulted in a $65 \%$ reduction in DNA content per well compared to control (Fig. 5A). The anti-proliferative effect was rescued to $59 \%$ of control when $32 \mu \mathrm{M}$ palmitic acid was supplied. Both the anti-proliferative and rescue effects were statistically significant when compared to control values ( $p<0.001$ and $<0.05$, respectively). Fibroblasts did not manifest an anti-proliferative response to CDDO-Me, and actually exhibited a significant $(\mathrm{p}<0.05)$ enhancement of growth compared to control when treated with palmitate alone, CDDO-Me alone, or both treatments combined (Fig. 5B). We speculate that this effect of CDDO-Me results from actions unrelated to lipid metabolism.

Using a non-quantitative assay, we previously reported that FASN and Spot 14 mRNA content was reduced in LiSa-2 cells treated with CDDO-Me (4). We now undertook quantitative real-time RT-PCR analysis of gene expression in LiSa-2 cells. This demonstrated an $86 \%$ decrease in FASN mRNA with triterpenoid treatment compared to vehicle control after 12 days in culture (Fig. 6A; p<0.01). Analysis of Spot 14 mRNA expression in these samples similarly showed a $60 \%$ decrease compared to control levels after 12 days of $100 \mathrm{nM}$ CDDO-Me treatment (Fig. 6C; $<<0.01$ ). In both experiments, levels of cyclophilin mRNA were not different $(\mathrm{p}=0.41)$ between treatment groups. In contrast to the 25 cycle RTPCR experiment depicted in Fig. 1, S14 and FAS mRNAs were detectable after 45 cycles of real-time RT-PCR in fibroblasts (Fig. 6B and D), and these did not vary in response to CDDO-Me $(\mathrm{p}=0.59)$. Thus, these results quantitatively confirm that CDDO-Me acts to decrease FASN and Spot 14 mRNAs in LiSa-2 cells. In addition, the fibroblasts were unaffected, further supporting the specificity of the growthinhibitory action of this compound on lipogenic sarcoma cells.

\section{Discussion}

Well-defined metabolic differences exist between malignant and non-malignant cells, particularly in glucose uptake and trapping, and in substrate utilization (19). Exploitation of these differences has been advocated as a potential therapeutic modality for malignancy (20). Fatty acids play an essential role in a variety of cellular processes. They are important components of plasma and organelle membranes, 
A

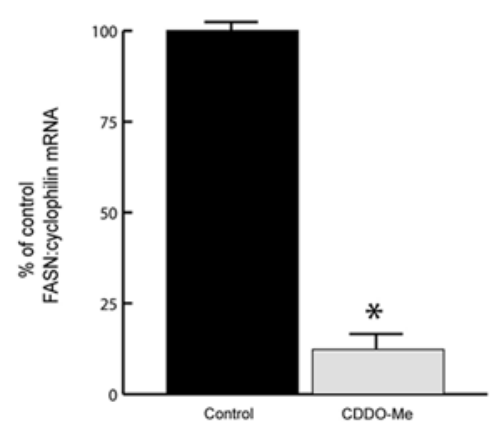

C

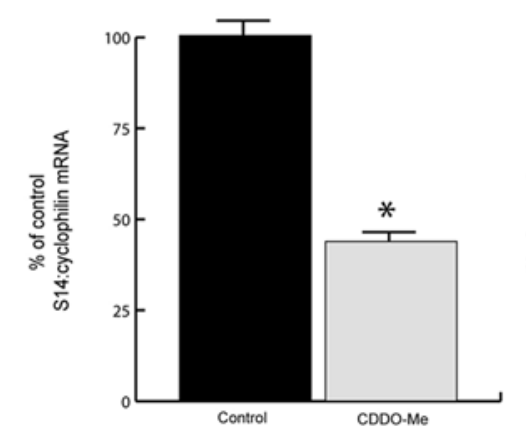

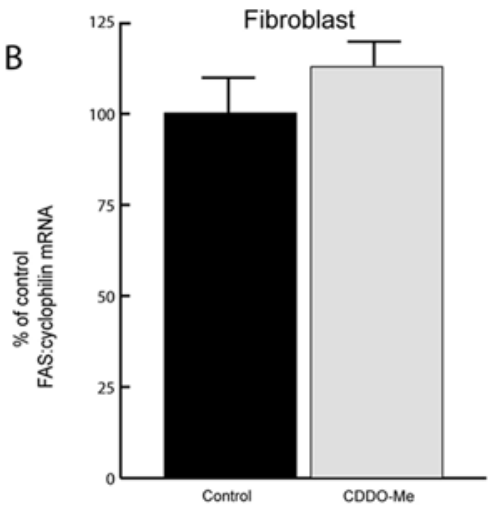

D

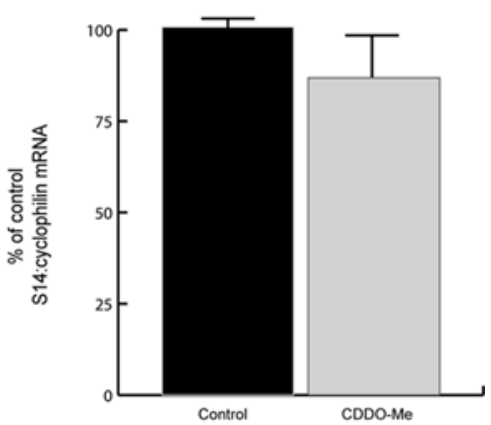

Figure 6. CDDO-Me downregulates FASN and S14 expression in LiSa-2 cells. Quantitative real-time PCR (45 cycles) results for fatty acid synthase (FASN) and S14 mRNAs in LiSa-2 (A and C) and fibroblast (B and D) cells after 12-day exposure to $100 \mathrm{nM}$ CDDO-Me or vehicle alone. Data (mean \pm SEM, $\mathrm{n}=6$ /group) are normalized to the cyclophilin signal and are expressed as a percent of control. * $\mathrm{p}<0.01$ compared to vehicle treatment.

they function in energy storage, and are oxidized as a fuel. In contrast to most normal cells, many cancer cells display a high rate of de novo fatty acid synthesis (21). Overexpression of FASN and Spot 14 has been linked to the lipogenic malignant phenotype that has been identified in a variety of common human cancers (8). This raises the possibility of therapy that takes advantage of the aberrant lipid metabolism of malignant cells. There is in vitro evidence that inhibition of FASN, ATP citrate lyase, or acetyl-CoA carboxylase offers therapeutic possibilities (12,22-25).

Global gene expression array analysis of human sarcoma histotypes indicated that many upregulated genes in LS are related to fatty acid metabolism. In comparison to other adult sarcomas, LS display an average of 5-fold overexpression of FASN mRNA (3). We recently reported that LiSa2 cells exhibit a pattern of gene expression similar to that of an adipocyte and, as expected, express key genes related to long-chain fatty acid synthesis, notably FASN and Spot 14 (4). Moreover, immunohistochemical analysis of LS tumor tissues confirmed the presence of the FASN and Spot 14 proteins. As in the case of other lipogenic cancer cells, liposarcoma cells also require a supply of fatty acids for growth and survival. In the current study we have shown similar patterns of lipogenic gene expression in two different human LS cell lines and provided evidence of a dependence on fatty acids for growth. Thus, using three chemically and mechanistically distinct inhibitors of lipogenic enzyme activities (cerulenin, orlistat, soraphen A), as well as a chemical inhibitor of FASN and Spot 14 gene expression (CDDO-Me), we demonstrated comparable inhibition of LS cell growth that is reversible by provision of palmitate. Moreover, in studies reported elsewhere, we observed similar suppression of FASN and Spot 14 gene expression in LS cells exposed to the $\mathrm{c} 9, \mathrm{t} 11$ - and $\mathrm{t} 10, \mathrm{c} 12$-isomers of the dietary supplement conjugated linoleic acid, which also inhibits growth of these cells (26).

These studies confirm the importance of fatty acids for growth and survival of liposarcoma cells. The rescue effect mediated by palmitate in the presence of FASN or ACC enzyme inhibition or reduced S14 and FASN gene expression underscores the significance of fatty acids and the genes related to their synthesis for LS cell growth. The prevention of cytotoxicity by palmitic acid, coupled with the similar antiproliferative effects of four structurally distinct compounds with differing mechanisms of action, provides strong evidence that it is the lack of fatty acids, rather than any other effects of these compounds, that mediates the growth inhibition. Moreover, none of these agents affects the growth of nonmalignant fibroblasts, indicating that the dependence on lipogenesis is specific to tumor cells.

The potential for targeting fatty acid synthesis in LS patients is intriguing, as the lipogenic pathway is not active in most tissues of individuals consuming a fatty Western diet (27). Theoretically, such anti-metabolic therapy should therefore not have significant effects on normal tissues except perhaps the cycling endometrium or the lactating breast. Moreover, the genes coding the major components of the fatty acid synthetic pathway are not redundant, and therefore 
alternate mechanisms for tumor lipogenesis are lacking. This has been shown by recent studies that have targeted other proteins required for de novo fatty acid synthesis, including ATP citrate lyase, ACC as in the current work, or Spot 14, demonstrating cytotoxic effects in each case (12,22-25).

Overall, targeting the fatty acid synthetic pathway offers the attractive clinical possibility of a medical approach to LS, which often recurs after attempted surgical cure, and upon recurrence is generally not controlled long-term by radiation and chemotherapy. These findings provide a foundation for further studies on the effects of these or similar agents in an in vivo pre-clinical model of liposarcoma.

\section{Acknowledgements}

We thank the following individuals for their contributions to this study: Eva Rzucidlo (Dartmouth Medical School) for the fibroblast cells, Michael Sporn (Dartmouth Medical School) for supplying synthetic triterpenoids, Rolf Jansen and Klaus Gerth (Helmholtz-Zentrum für Infektionsforschung, Braunschweig, Germany) for providing soraphen A, and Martin Wabitsch (University of Ulm, Germany) for the LiSa-2 cells. This work was supported by N.I.H. grant RO1CA126618 (to WBK), a Norris Cotton Cancer Center Prouty grant (to BLE), N.I.H. Training Grant DK07508 (to NBK), a Howard Hughes Fellowship (to AJF), and a grant from the Research Foundation-Flanders (FWO) (to JVS).

\section{References}

1. Mack T: Sarcomas and other malignancies of soft tissue, retroperotineum, peritoneum, pleura, heart, mediastinum, and spleen. Cancer 75: S211-S244, 1995.

2. Patel S, Burgess M, Plager C, Papadopoulos N, Linke K and Benjamin R: Myxoid liposarcoma: experience with chemotherapy. Cancer 74: 1265-1269, 1994.

3. Baird K, Davis S, Antonescu C, et al: Gene expression profiling of human sarcomas: insights into sarcoma biology. Cancer Res 65: 9226, 2005.

4. Hughes D, Martel P, Kinlaw W and Eisenberg B: The synthetic triterpenoid CDDO-Im inhibits fatty acid synthase expression and has antiproliferative and proapoptotic effects in human liposarcoma cells. Cancer Invest 26: 118-127, 2007.

5. Menendez J and Lupu R: Fatty acid synthase and the lipogenic phenotype in cancer pathogenesis. Nat Rev Cancer 7: 763-777, 2007.

6. Wells W, Schwartz G, Morganelli P, Cole B, Chambers J and Kinlaw WB: Expression of 'Spot 14' (THRSP) predicts disease free survival in invasive breast cancer: immunohistochemical analysis of a new molecular marker. Breast Cancer Res Treat 98: 231-240, 2006.

7. Martel P, Bingham C, McGraw C, et al: S14 protein in breast cancer cells: direct evidence for regulation by SREBP-1c, superinduction with progestin, and implication in cell growth. Exp Cell Res 312: 278-288, 2005.

8. Kinlaw W, Quinn J, Wells W, Roser-Jones C and Moncur J: S14 in breast cancer: a marker of aggressive disease and a potential therapeutic target. Endocrinology 147: 4048-4055, 2006.
9. Pizer E, Jackisch C, Wood F, Pasternak G, Davidson N and Kuhajda F: Inhibition of fatty acid synthesis induces programmed cell death in human breast cancer cells. Cancer Res 56: 2745-2747, 1996.

10. Menendez J, Vellon L and Lupu R: Antitumoral actions of the anti-obesity drug orlistat (Xenical) in breast cancer cells: blockade of cell cycle progression, promotion of apoptotic cell death and PEA3-mediated transcriptional repression of Her2/ neu (erbB-2) oncogene. Ann Oncol 16: 1253-1267, 2005.

11. Kridel S, Axelrod F, Rozenkrantz N and Smith J: Orlistat is a novel inhibitor of fatty acid synthase with antitumor activity. Cancer Res 64: 2070-2075, 2004.

12. Beckers A, Organe S, Timmermans L, et al: Chemical inhibition of acteyl-CoA carboxylase induces growth arrest and cytotoxicity selectively in cancer cells. Cancer Res 67: 8180-8187, 2007.

13. Suh N, Wang Y, Honda T, et al: A novel synthetic oleanane triterpenoid, 2-cyano-3,12-dioxoolean-1,9-dien-28-oic acid, with potent differentiating, antiproliferative, and anti-inflammatory activity. Cancer Res 59: 336-341, 1999.

14. Ip M, Masso-Welch P, Shoemaker S, Shea-Elton W and Ip C: Conjugated linoleic acid inhibits proliferation and induces apoptosis of normal rat mammary epithelial cells in primary culture. Exp Cell Res 250: 22-34, 1999.

15. Wabitsch M, Bruderlein S, Melzner I, Braun M, Mechterscheimer G and Moller P: LiSa-2, a novel human liposarcoma cell line with a high capacity for terminal adipose differentiation. Int J Cancer 88: 889-894, 2000.

16. Bligh E and Dyer W: A rapid method of total lipid extraction and purification. Can J Biochem Physiol 37: 911-917, 1959.

17. Waddell WJ: A simple ultraviolet spectrophotometric method for the determination of protein. J Lab Clin Med 48: 311-315, 1956.

18. Rago R, Mitchen $\mathbf{J}$ and Wilding G: DNA fluorometric assay in 96-well tissue culture plates using Hoechst 33258 after cell lysis by freezing in distilled water. Anal Biochem 191: 31-34, 1990.

19. Menendez J and Lupu R: Oncogenic properties of endogenous fatty acid synthesis: molecular pathology of fatty acid synthase in cancer cells. Curr Opin Nutr Metab Care 9: 346-357, 2006.

20. Swinnen J, Brusselmans K and Verhoeven G: Increased lipogenesis in cancer cells: new players, novel targets. Curr Opin Clin Nutr Metab Care 9: 358-365, 2006.

21. Kuhajda F: Fatty-acid synthase and human cancer: New perspectives on its role in tumor biology. Nutrition 16: 202-208, 2000.

22. Kuhajda F: Fatty acid synthase and cancer: new application of an old pathway. Cancer Res 66: 5977-5980, 2006.

23. Chajes V, Cambot M, Moreau K, Lenoir G and Joulin V: Acetyl-CoA carboxylase-a is essential to breast cancer cell survival. Cancer Res 66: 5287-5294, 2006.

24. Hatsivassiliou G, Zhao F, Bauer D, et al: ATP citrate lyase inhibition can suppress tumor cell growth. Cancer Cell 8: 311-321, 2005.

25. Brusselmans K, De Schrijver E, Verhoeven G and Swinnen J: RNA interference-mediated silencing of the acetyl-CoAcarboxylase-a gene induces growth inhibition and apoptosis of prostate cancer cells. Cancer Res 65: 6719-6725, 2005.

26. Donnelly C, Olsen A, Lewis L, Eisenberg B, Eastman A and Kinlaw W: Conjugated linoleic acid (CLA) inhibits expression of the Spot 14 (THRSP) and fatty acid synthase genes and impairs the growth of human breast cancer and liposarcoma cells. Nutr Cancer 61: 114-122, 2009.

27. Weiss L, Hoffman G, Schreiber R, Andres H, Fuchs E, Korber E and Kolb H: Fatty-acid biosynthesis in man, a pathway of minor importance. J Biol Chem 367: 905-912, 1986. 